\title{
Correction to: Post-induction MRD by FCM and GATA1-PCR are significant prognostic factors for myeloid leukemia of Down syndrome
}

Takashi Taga (D), Shiro Tanaka, Daisuke Hasegawa (D), Kiminori Terui, Tsutomu Toki (D), Shotaro Iwamoto (iD, Hidefumi Hiramatsu (D), Takako Miyamura (D), Yoshiko Hashii, Hiroshi Moritake (D), Hideki Nakayama (D), Hiroyuki Takahashi (D), Akira Shimada, Tomohiko Taki, Etsuro Ito (D), Asahito Hama (iD, Masafumi Ito, Katsuyoshi Koh (iD, Daiichiro Hasegawa, Akiko M. Saito, Souichi Adachi and Daisuke Tomizawa (D)

(C) The Author(s), under exclusive licence to Springer Nature Limited 2021

Leukemia (2021) 35:3622-3624; https://doi.org/10.1038/s41375-021-01397-w

Correction to: Leukemia https://doi.org/10.1038/s41375-02101157-w, published online 15 February 2021

Following the publication of this article, the authors noted an error in the data reported.

One patient in the AML-D11 study (showing negativity for both FCM-MRD and GATA1-MRD) was incorrectly reported dead after relapse following a data input error. After re-confirming the survival data for all other patients, the dataset has been updated and results are as follows.

- The number of patients who relapsed was decreased from 7 to 6 .

- The 3-year event-free survival (EFS) and overall survival (OS) rates in the entire population $(n=78)$ were 88.5 and $91.0 \%$.

- The 3-year EFS and OS rates in the SR patients $(n=76)$ were 90.8 and $93.4 \%$.

- The 3-year EFS and OS rates were 95.0 and $96.7 \%$ in the FCM-
MRD-negative population, and 60.0 and $80.0 \%$ in the positive population.

- Three-year EFS and OS rates were both $98.1 \%$ in the GATA1MRD-negative population, and 57.1 and $71.4 \%$ in the positive population.

- The adjusted hazard ratios for the association of FCM-MRD with EFS and OS were $14.67(95 \% \mathrm{Cl}, 2.00-107.79 ; p=0.01)$ and $13.48(0.81-224.27 ; p=0.07)$, respectively, while multivariate analysis of GATA1-MRD did not converge in the updated dataset due to the limited number of events in the GATA1-MRD-negative population.

Table 2, Supplementary Table 4, and Figs. 3 and 4 have been corrected and are shown below.

The conclusions of the article remain unchanged.

Table 2. Multivariate Cox regression of FCM-MRD for event-free and overall survivals in the standard risk population.

\begin{tabular}{|c|c|c|c|c|c|c|}
\hline & \multicolumn{3}{|l|}{ EFS } & \multicolumn{3}{|l|}{ os } \\
\hline & HR & $95 \% \mathrm{Cl}$ & $\boldsymbol{p}$ & HR & $95 \% \mathrm{Cl}$ & $p$ \\
\hline \multicolumn{7}{|c|}{ Analysis of FCM-MRD ( $N=65$, including five MRD-positive patients) } \\
\hline Negative & Ref & & & Ref & & \\
\hline Positive & 14.67 & $2.00-107.79$ & 0.01 & 13.48 & $0.81-224.27$ & 0.07 \\
\hline \multicolumn{7}{|l|}{ Cytogenetics } \\
\hline $1 q$ loss & 6.03 & $0.53-69.21$ & 0.15 & 10.68 & $0.67-170.88$ & 0.09 \\
\hline Platelet (per $10^{10} / \mathrm{L}$ increase) & 1.09 & $0.87-1.37$ & 0.45 & 0.95 & $0.65-1.37$ & 0.77 \\
\hline
\end{tabular}

MRD minimal residual disease, EFS event-free survival, $O S$ overall survival, $H R$ hazard ratio, $C l$ confidence interval.

Multivariate analysis of GATA1-MRD did not converge due to the limited number of events in the GATA1-MRD-negative population. 
Table S4. Univariate Cox regression of prognostic factors for event-free and overall survivals among 78 patients.

\begin{tabular}{|c|c|c|c|c|c|c|}
\hline & EFS & & & os & & \\
\hline & HR & $95 \% \mathrm{Cl}$ & $p$ & HR & $95 \% \mathrm{Cl}$ & $\boldsymbol{P}$ \\
\hline Age at diagnosis $\geq 24$ months & 4.71 & $1.05-21.07$ & 0.04 & 5.28 & $0.88-31.61$ & 0.07 \\
\hline Mosaic 21 trisomy & 3.56 & $0.65-19.42$ & 0.14 & 7.32 & $1.03-52.05$ & 0.05 \\
\hline History of TAM & 0.52 & $0.12-2.31$ & 0.39 & 0.45 & $0.08-2.72$ & 0.39 \\
\hline Cardiac complication & 0.71 & $0.13-3.90$ & 0.70 & 0.36 & $0.05-2.55$ & 0.31 \\
\hline M1 & 18.24 & $2.04-163.20$ & 0.01 & Not estimable & & \\
\hline M7 & 1.68 & $0.38-7.49$ & 0.50 & 3.51 & $0.59-20.99$ & 0.17 \\
\hline RAEB-T & 1.28 & $0.25-6.57$ & 0.77 & 2.10 & $0.35-12.60$ & 0.41 \\
\hline RAEB & 0.23 & $0.03-1.95$ & 0.18 & Not estimable & & \\
\hline RA & Not estimable & & & Not estimable & & \\
\hline \multicolumn{7}{|l|}{ Cytogenetics } \\
\hline Normal karyotype & 0.54 & $0.06-4.60$ & 0.57 & Not estimable & & \\
\hline Monosomy 7 & Not estimable & & & Not estimable & & \\
\hline Monosomy 7 (complex) & 1.92 & $0.22-16.47$ & 0.55 & 3.59 & $0.37-34.55$ & 0.27 \\
\hline Sole trisomy 8 & Not estimable & & & Not estimable & & \\
\hline Complex & 1.78 & $0.21-15.23$ & 0.60 & 2.83 & $0.29-27.23$ & 0.37 \\
\hline $1 \mathrm{q}$ loss & 6.11 & $1.12-33.39$ & 0.04 & 12.41 & $1.75-88.28$ & 0.01 \\
\hline $7 p$ loss & 1.35 & $0.16-11.60$ & 0.78 & 2.18 & $0.23-20.97$ & 0.50 \\
\hline Acquired +21 & Not estimable & & & Not estimable & & \\
\hline Others & 1.44 & $0.29-7.15$ & 0.65 & 1.41 & $0.20-10.01$ & 0.73 \\
\hline
\end{tabular}

EFS event-free survival, OS overall survival, HR hazard ratio, $\mathrm{Cl}$ confidence interval. 
(a)

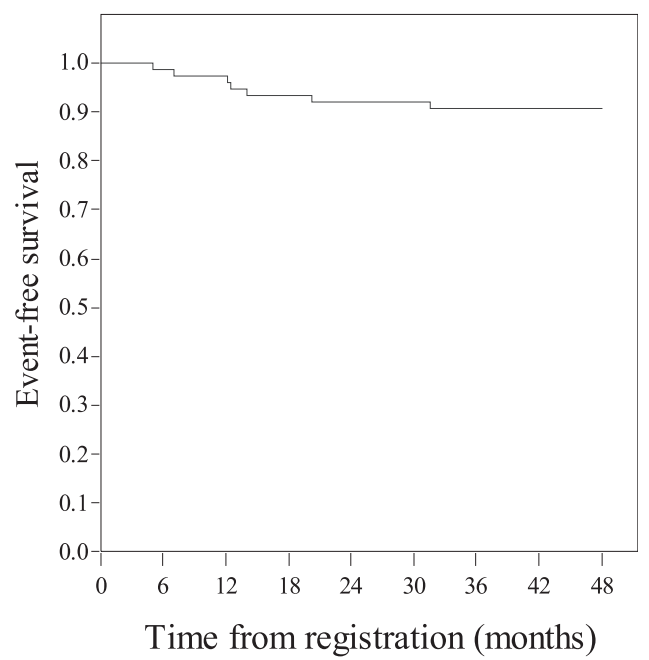

No. at Risk Standard risk

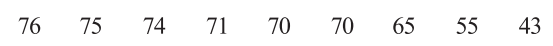

(b)

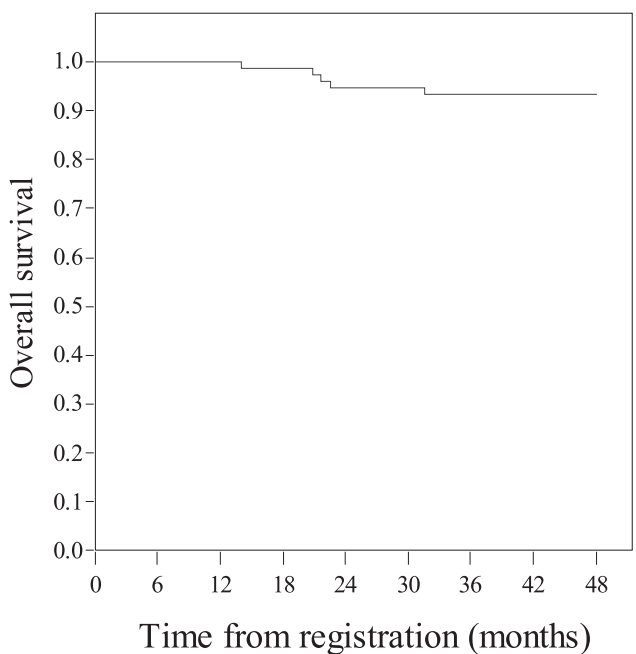

No. at Risk Standard risk $\begin{array}{lllllllll}76 & 76 & 76 & 75 & 72 & 72 & 67 & 55 & 43\end{array}$ (a)

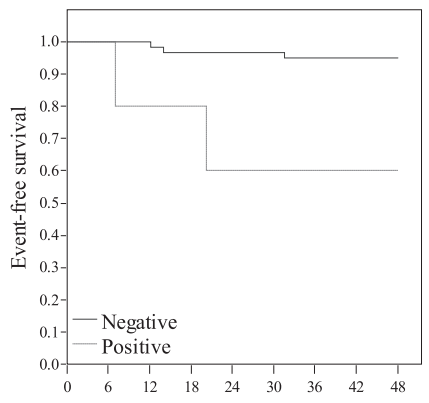

Time from registration (months)

No. at Risk Negative
Positive

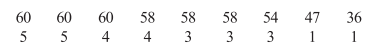

(b)

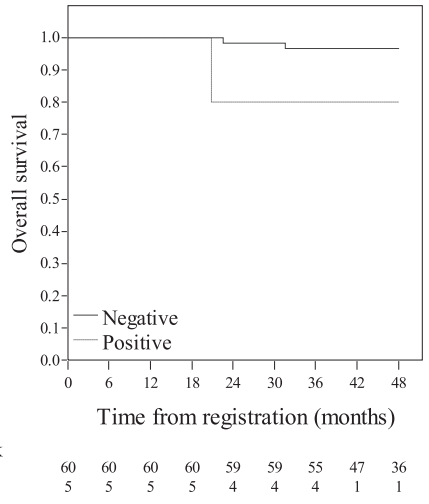

(c)

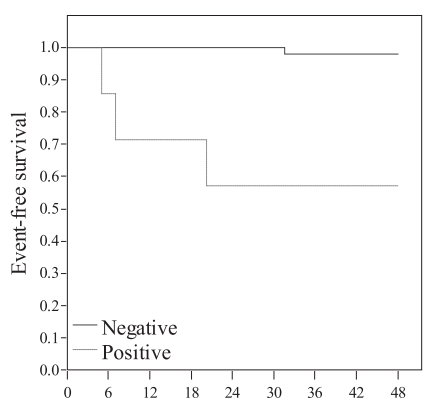

Time from registration (months)

No. at Risk Negative
Positive

(d)
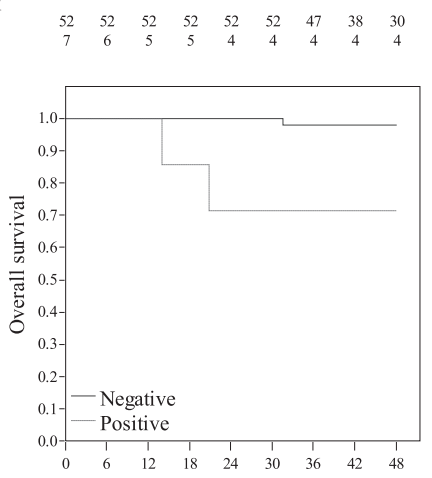

Time from registration (months)

No. at Risk

Negative

$\begin{array}{ccccccccc}52 & 52 & 52 & 52 & 52 & 52 & 47 & 38 & 30 \\ 7 & 7 & 7 & 6 & 5 & 5 & 5 & 4 & 4\end{array}$ 